\title{
Morfología de los óxidos superficiales en los recubrimientos convencionales y nanocristalinos de NiCrAlY sometidos a alta temperatura
}

\author{
J.A. PICAS ${ }^{1}$, A. FORN 1 , L. AJDELSZTAJN 2 , J. SCHOENUNG ${ }^{2}$ \\ ${ }^{1}$ Departamento de Ciencia de Materiales, Universitat Politècnica de Catalunya, Vilanova i la Geltrú, 08800, España. E \\ ${ }^{2}$ Department of Chemical Engineering and Materials Science, University of California, Irvine, CA 92697-2575, USA.
}

\begin{abstract}
El objetivo del presente trabajo es el estudio de la morfología de los óxidos formados en la superficie de los recubrimientos convencionales y nanocristalinos de NiCrAlY, obtenidos mediante proyección térmica $\mathrm{HVOF}$, al someter el material a $1000^{\circ} \mathrm{C}$. El afino de grano obtenido en el recubrimiento nanocristalino de NiCrAlY parece ser el responsable de la variación de la morfología de los óxidos formados después del tratamiento a alta temperatura, lo cual sugiere que podrán presentar un mejor comportamiento frente a la oxidación en comparación con los recubrimientos convencionales.
\end{abstract}

Palabras Clave: Proyección térmica HVOF; recubrimientos de NiCrAlY; oxidación; morfología.

\section{Morphology of the superficial oxides in conventional and nanocrystalline NiCrAlY coatings exposed to elevated temperature}

HVOF thermal spray process was used to produce conventional and nanostructured NiCrAlY coatings. The oxidation behaviour at $1000^{\circ} \mathrm{C}$ of both coatings has been studied. The morphology of the oxides suggests that the nanostructured coating exhibited improved oxidation behaviour compared to conventional counterparts.

Keywords: HVOF thermal spray; NiCrAlY coatings; oxidation ; morphology.

\section{INTRODUCCIÓN}

Para proteger los álabes de las turbinas en los motores de reacción se emplean recubrimientos multicapas que actúan como barreras térmicas (Thermal Barrier Coatings - TBC), los cuales están constituidos habitualmente por una primera capa metálica de NiCrAlY y un recubrimiento cerámico superficial de $\mathrm{ZrO}_{2} / \mathrm{Y}_{2} \mathrm{O}_{3}$. La capa de óxido formada térmicamente en la superficie del recubrimiento de NiCrAlY, puede jugar un papel crítico en la aparición de grietas en la interfase entre ambos recubrimientos, favoreciendo el desprendimiento prematuro del recubrimiento cerámico $(1,2)$.

Los recubrimientos de NiCrAlY se obtienen usualmente mediante procesos de proyección térmica por plasma (Vacuum Plasma Spray - VPS o Atmospheric Plasma Spray - APS) o más recientemente mediante procesos de proyección térmica HVOF (High Velocity Oxy Fuel) $(3,4)$. El proceso HVOF se caracteriza por una alta velocidad de las partículas depositadas así como por una temperatura relativamente baja $(2000 \mathrm{~K})$ en comparación con otras técnicas de proyección térmica, por lo que puede emplearse esta técnica en la obtención de recubrimientos nanocristalinos $(5,6)$.

\section{PARTE EXPERIMTENTAL}

Los recubrimientos convencionales y nanocrisatlinos de $\mathrm{Ni}-22 \mathrm{Cr}-$ 10Al-1Y (\% en peso) se han obtenido mediante proyección térmica HVOF sobre un substrato de níquel, utilizando un equipo Sulzer Metco Diamond Jet DJ 2700 HVOF Thermal Spray (7). En la tabla I se muestran los parámetros de proyección.

Para la obtención de los recubrimientos se partió de polvos comerciales fabricados por la empresa Praxair Surface Technologies - Tafa (Ni-343), los cuales fueron triturados mecánicamente en un molino de bolas con la finalidad de obtener polvos con características nanocris- talinas. El proceso de molienda se realizó en condiciones criogénicas, mediante la inmersión en nitrógeno líquido, durante 12 horas y a una velocidad de giro de $180 \mathrm{rpm}$, empleando bolas de acero de $0.635 \mathrm{~cm}$ de diámetro y una relación en peso partículas - bolas de 1:30 $(6,7)$.

TABLA I: PARÁMETROS DE PROYECCIÓN USADOS PARA PRODUCIR LOS RECUBRIMIENTOS DE NICRALY

\begin{tabular}{|l|c|c|c|c|}
\hline Gas & $\begin{array}{c}\text { Presión } \\
(\mathrm{MPa})\end{array}$ & $\begin{array}{c}\text { flujo de gas } \\
\left(\mathrm{mm}^{3} / \mathrm{s}\right)\end{array}$ & Parámetros & Ajuste \\
\hline Aire & 0.69 & 5616 & $\begin{array}{c}\text { Velocidad de aportación } \\
\text { del polvo }\end{array}$ & $0.315 \mathrm{~g} / \mathrm{s}$ \\
\hline $\begin{array}{l}\text { Combustible } \\
\text { (Propileno) }\end{array}$ & 0.69 & 1488 & $\begin{array}{c}\text { Velocidad transversal de } \\
\text { la pistola de proyección }\end{array}$ & $1.1016 \mathrm{~m} / \mathrm{s}$ \\
\hline Oxígeno & 1.034 & 3410 & Distancia de proyección & $0.230 \mathrm{~m}$ \\
\hline
\end{tabular}

Después del deposito de los recubrimientos, se llevaron a cabo los experimentos de oxidación en aire a una temperatura de $1000{ }^{\circ} \mathrm{C}$ y diferentes tiempos (2, 4, 12, 24 y 48 horas). La identificación de los óxidos formados en la superficie se realizó por difracción de rayos X (difractómetro Siemens D500) y el estudio de su morfología fue realizado mediante microscopía electrónica de barrido (Philips XL30 FEG).

\section{RESULTADOS Y DISCUSION}

Mediante difracción de rayos $\mathrm{X}$ se han identificado las fases que constituyen los polvos de NiCrAlY, que están constituidos principalmente por dos fases: fase $\gamma$ (solución sólida de $\mathrm{Ni}$ con $\mathrm{Cr}$ ) y fase $\gamma^{\prime}$ 
(compuesto intermetalico $\mathrm{Ni}_{3} \mathrm{Al}$ ). Después de 12 horas de molienda el espectro de difracción de rayos $\mathrm{X}$ presenta el ensanchamiento de los picos de las fases $\gamma$ y $\gamma^{\prime}$, como consecuencia del pequeño tamaño de grano obtenido (valor medio del tamaño de grano: $26 \mathrm{~nm}$ ) (8). Una vez depositados los polvos mediante HVOF, la distribución del tamaño de grano en el recubrimiento convencional es relativamente homogénea, con un tamaño de grano entre 1 y $1,5 \mu \mathrm{m}$, mientras que en el recubrimiento nanocristalino se observa, mediante microscopía electrónica de transmisión, que el recubrimiento presenta un tamaño de grano que se extiende desde regiones nanocristalinas de aproximadamente $75 \mathrm{~nm}$ a regiones con tamaños de grano mayores (de hasta $550 \mathrm{~nm}$ ).

Después de 24 horas de tratamiento térmico a $1000^{\circ} \mathrm{C}$, en la superficie de los recubrimientos se observa la formación de una capa de óxido (figura 1). Esta capa es continua y homogénea en los recubrimientos nanocristalinos (figura 1a), mientras que en los recubrimientos convencionales aparece discontinua y no uniforme, observándose la formación de protuberancias en la superficie del material (figura 1b). De acuerdo con los resultados de difracción de rayos $\mathrm{X}$, los productos de oxidación presentes en los recubrimientos convencionales son $\alpha-\mathrm{Al}_{2} \mathrm{O}_{3}$ y $\mathrm{NiO}$, así como pequeñas cantidades de otras fases tipo espinela $\left(\mathrm{NiAl}_{2} \mathrm{O}_{4} \mathrm{y}\right.$ $\mathrm{NiCr}_{2} \mathrm{O}_{4}$ ). En contraste, en los recubrimientos nanocristalinos el principal óxido presente corresponde a la formación de $\alpha-\mathrm{Al}_{2} \mathrm{O}_{3}$

En la figura 2a se puede observar la formación de las diferentes morfologías de los óxidos superficiales formados en los recubrimien-

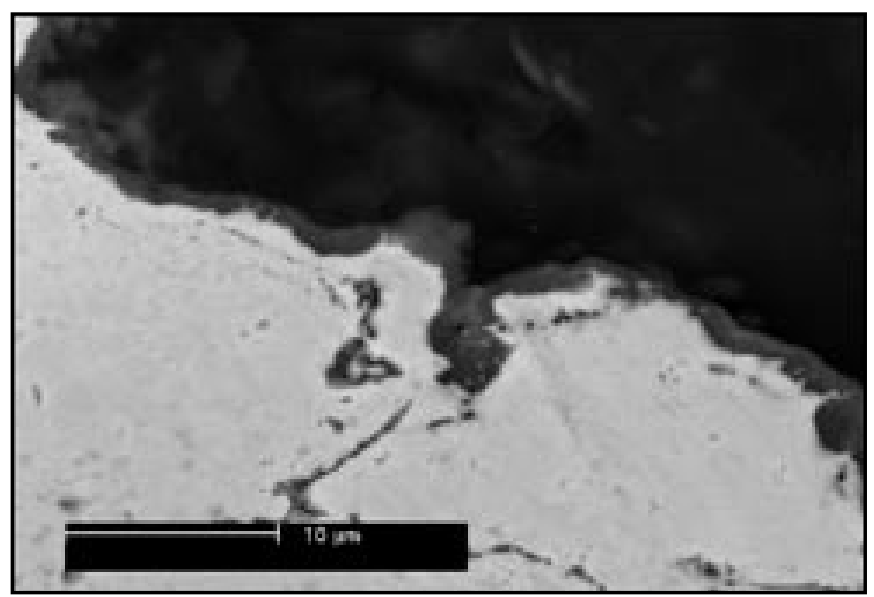

(a)

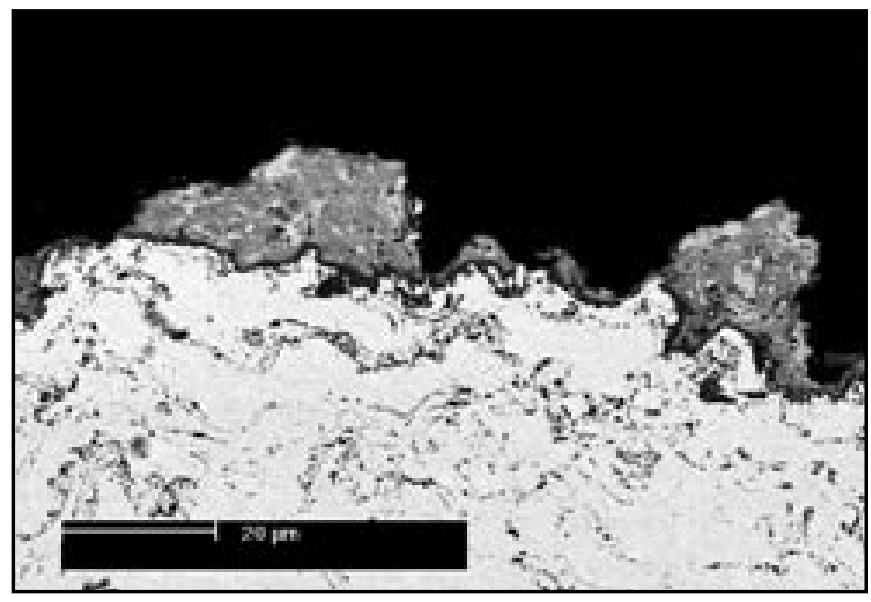

(b)

Figura 1: Formación de una capa de óxido en la superficie de los recubrimientos de NiCrAlY nanocristalinos (a) y convencionales (b) después del tratamiento de oxidación a $1000{ }^{\circ} \mathrm{C}$ durante 24 horas tos convencionales después del tratamiento térmico a $1000{ }^{\circ} \mathrm{C}$ durante 48 horas. La estructura uniforme en forma de crestas, de acuerdo con los análisis por EDS (Espectrometría de Energía Dispersada de rayos $\mathrm{X}$ ), corresponde con la típica estructura de la fase $\alpha-\mathrm{Al}_{2} \mathrm{O}_{3^{\prime}}$ mientras que los cristales poliédricos corresponden a la estructura de $\mathrm{NiO}$ (4). La figura $2 \mathrm{~b}$ muestra un detalle de morfología de los cristales de $\mathrm{NiO}$, con una forma octaédrica definida por planos del tipo $\{111\}$ (estructura tipo $\mathrm{NaCl}$ ).

En contraste, la morfología de la superficie observada en los recubrimientos nanocristalinos presenta la formación de una estructura de $\alpha-\mathrm{Al}_{2} \mathrm{O}_{3}$ uniformemente distribuida por toda la superficie (figura $3 \mathrm{a}$ ). La figura $3 \mathrm{~b}$ muestra un detalle de la estructura característica de la fase $\alpha-\mathrm{Al}_{2} \mathrm{O}_{3}$ (estructura cristalina tipo corindón) definida por el crecimiento de los planos $\{0001\}$.

La morfología y composición de la capa de óxido superficial pueden ser críticas en el comportamiento de los recubrimientos de $\mathrm{Ni}$ CrAlY que se emplean en la fabricación de barreras térmicas. La mayor velocidad de difusión del aluminio a través de las fronteras de grano en los recubrimientos nanocristalinos parece favorecer la formación de una capa de alúmina continua y homogénea que evita la formación de protuberancias superficiales. La presencia de estas protuberancias en los recubrimientos convencionales, comporta un aumento considerable del volumen de la capa de óxido $(\mathrm{NiO})$ que consecuentemente afectará la vida del posible recubrimiento cerámico exterior.

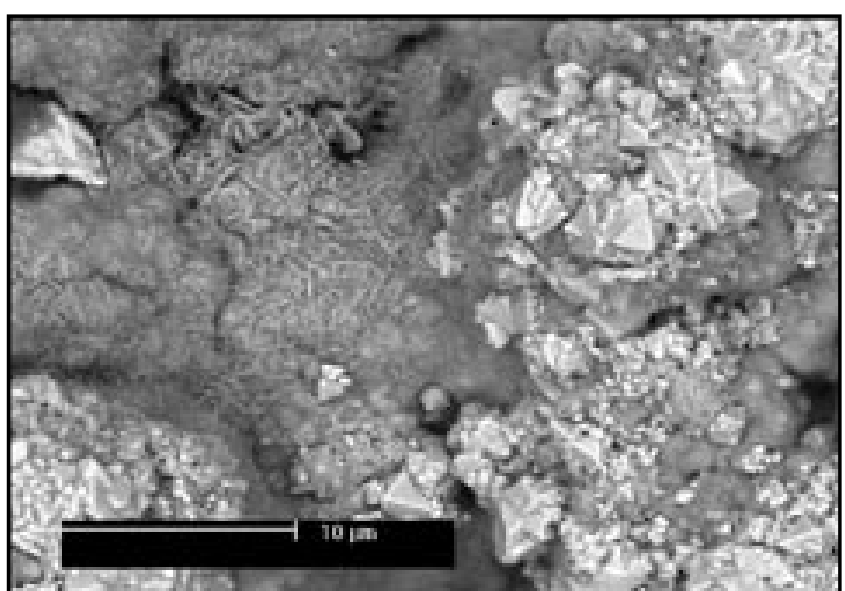

(a)

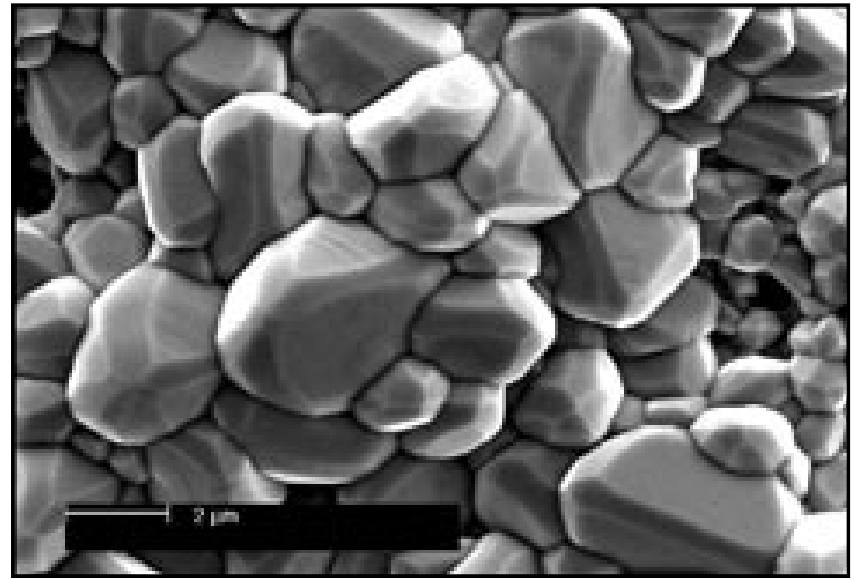

(b)

Figura 2: (a) Morfología de los óxidos formados en la superficie del recubrimiento de NiCrAlY convencional después de 48 horas de tratamiento térmico. (b) detalle de los cristales octaédricos de $\mathrm{NiO}$. 


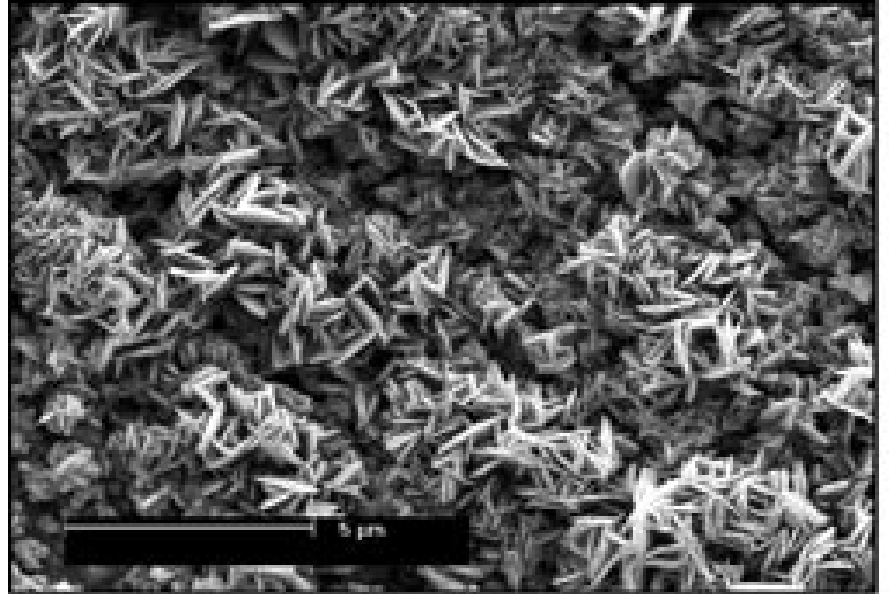

(a)

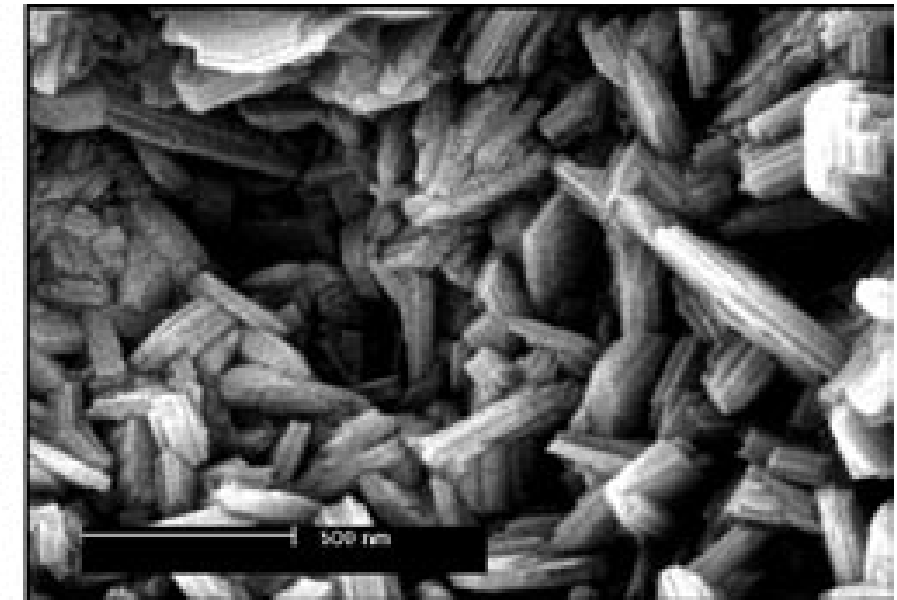

(b)

Figura 3: (a) Morfología de los óxidos formados en la superficie del recubrimiento de NiCrAlY nanocristalino después de 48 horas de tratamiento térmico. (b) detalle de los cristales de $\alpha-\mathrm{Al}_{2} \mathrm{O}_{3}$.

\section{CONCLUSIONES}

El proceso de trituración mecánica en condiciones criogénicas de los polvos comerciales de NiCrAlY ha permitido obtener polvos de $\mathrm{Ni}$ CrAlY con características nanocristalinas. Mediante el proceso HVOF, se han obtenido los correspondientes recubrimientos de NiCrAlY convencionales y nanocristalinos.

La disminución del grano obtenido en los recubrimientos de NiCrAlY es el responsable de la variación de la morfología de los óxidos formados en la superficie del material después del tratamiento a 1000 ${ }^{\circ} \mathrm{C}$. Los recubrimientos convencionales presentan una capa de alúmina discontinua y la formación de protuberancias constituidas por $\mathrm{NiO}$ y espinelas $\mathrm{Ni}(\mathrm{Cr}, \mathrm{Al})_{2} \mathrm{O}_{4^{\prime}}$ mientras que en los recubrimientos nanocristalinos se ha observado la formación de una capa continua y homogénea de $\alpha-\mathrm{Al}_{2} \mathrm{O}_{3}$.

Los recubrimientos de NiCrAlY nanocristalinos, obtenidos por HVOF, podrán presentar una mejor resistencia a la oxidación en comparación con los recubrimientos convencionales y consecuentemente permitirán una mayor estabilidad de los recubrimientos multicapas que actúan como barreras térmicas.

\section{BIBLIOGRAFIA}

1. J.T DeMasi, K.D Sheffler, S. Bose, J. of eng. for gas turbine engines and power, 112, 521-526, 1990.

2. R.V. Hillery, B.H. Pilsner, R.L. McKnight, T.S. Cook, M.S. Hartle, Thermal Barrier Coating Life Prediction Model Development, NASA CR 180807, NASA, Washington, DC, Nov. 1988.

3. W. Brandl, D. Toma, J. Kruger, H.J. Grabke, G. Matthaus. "The oxidation behavoiur of HVOF thermal-sprayed MCrAlY coatings", Surface and Coatings Technology, 94-95, 21-26, 1997.

4. D. Toma, W. Brandl, U. Koster. Studies on the transient stage of oxidation of VPS and HVOF sprayed MCrAlY coatings, Surface and Coatings Technology, 120, 8-15, 1999

5. H.G. Jiang, M.L. Lau, V.L. Tellkamp, and E.J. Lavernia. Handbook of Nanostructured Materials and Nanotechnology: Synthesis and Processing, H. S. Nalwa, Ed., Academic Press, 159-213, 2000.

6. M.L. Lau, H.G. Jiang, W. Nuechter, E.J. Lavernia. “Thermal spraying of nanocrystalline Ni coatings". Phys. Status Solidi A, 166, 1, 257-268, 1998.

7. J. He, M. Ice, and E. J. Lavernia. "Synthesis and processing of nanostructured $\mathrm{Cr}$ sub 3C sub 2-NiCr coatings using mechanical milling and HVOF. Materials science Forum", 312, 237-246, 1999.

8. H.P. Klug, L.E. Alexander. X-ray Diffraction Procedures, New York, NY: John Wiley\&Sons, 1974.

Recibido: 1.2.03

Aceptado: 30.11 .03 